This item was submitted to Loughborough's Research Repository by the author.

Items in Figshare are protected by copyright, with all rights reserved, unless otherwise indicated.

\title{
Interface pressure and the prediction of car seat discomfort
}

PLEASE CITE THE PUBLISHED VERSION

http://dx.doi.org/10.1016/S0003-6870(98)00018-0

\section{PUBLISHER}

(c) Elsevier

VERSION

AM (Accepted Manuscript)

\section{PUBLISHER STATEMENT}

This work is made available according to the conditions of the Creative Commons Attribution-NonCommercialNoDerivatives 4.0 International (CC BY-NC-ND 4.0) licence. Full details of this licence are available at: https://creativecommons.org/licenses/by-nc-nd/4.0/

\section{LICENCE}

CC BY-NC-ND 4.0

\section{REPOSITORY RECORD}

Gyi, Diane E., and J. Mark Porter. 2019. "Interface Pressure and the Prediction of Car Seat Discomfort". figshare. https://hdl.handle.net/2134/21067. 
Gyi, D.E. and Porter J.M. (1999). Interface pressure and the prediction of car seat discomfort. Applied Ergonomics 30, 99-107.

\title{
Interface pressure and the prediction of car seat discomfort
}

\author{
Diane E. Gyi and J. Mark Porter \\ Vehicle Ergonomics Group, Department of Design and Technology, Loughborough \\ University, Leicestershire, LE11 3TU, UK. Tel: 01509 223043, Fax: 01509223999
}

\begin{abstract}
The technique of interface pressure measurement has generated considerable interest in the automotive industry as a method, which could be used to predict driver discomfort during the development of prototype seat designs. Two repeated measures experiments were carried out to evaluate the practical application of the technique. The variables of foam density and posture were used to create discomfort, the whole emphasis of the work being to generate results with real world applicability. A clear, simple and consistent relationship between interface pressure and driving discomfort was not identified. Future studies using this technique should provide information regarding such factors as gender, body mass, anthropometric data, posture and foam hardness due to the confounding nature of these variables.
\end{abstract}

Keywords: driving, interface pressure measurement, discomfort, car seating

\section{Introduction}

There are high hopes in the automotive industry that seat interface pressure measurement can be used to predict areas of subjective discomfort. The Vehicle Ergonomics Group (VEG) have established methods for evaluating the driver's workstation (seat comfort, vision, reach) using subjective discomfort data. Whilst these data are of high quality, road trials may take several months to complete and are often carried out when the car is almost ready for production (Porter, 1995). Predictive techniques would provide car seat designers and manufacturers with rapid information early on in the design process, which 
Gyi, D.E. and Porter J.M. (1999). Interface pressure and the prediction of car seat discomfort. Applied Ergonomics 30, 99-107.

could be passed on to the consumer in the form of high quality seating systems minimising discomfort.

Few studies have yet attempted to correlate seat comfort or driver discomfort with interface pressure. In the study by Kamijo et al (1982), 43 car seats were evaluated as being comfortable or uncomfortable, although the time duration of the evaluation was not indicated. Their results stated that static pressure distribution 'approximately correlated' with the difference between comfortable and uncomfortable seats. However the analyses were based on the patterns of pressure readings of only one subject being matched with the subjective evaluations of each seat by the 15 subjects.

Lee and Ferraiuolo (1993) used 100 subjects in their experiment evaluating 16 visually similar car seats. The seats were fabricated from production ranges by varying the parameters of foam thickness and hardness, back contour and angle, cushion angle, spring suspension rates and side support. Subjects sat in each seat for a minimum of only two minutes and assessed their perceived comfort in ten body areas. Despite the large number of subjects, the authors concluded that there were not enough correlations between pressure and subjective comfort to form the basis of design decisions.

Gross et al (1994) recorded the perceived comfort of 12 aspects of the seat for each of 50 car seats (more than 1100 seat-subject combinations), each seat trial lasting 5-10 minutes. The authors concluded that the pressure data statistics were strongly related to perceived comfort and therefore perceived comfort could be predicted. However, no details were given regarding the statistical analysis and the fact that the relationship between pressure and perceived comfort was only based on a 5-10 minute assessment limits its value.

Shen and Galer (1993) attempted to build a multi-factor model of sitting discomfort using interface pressure measurements. Based on their literature review they identified 'the force applied to the body', 'the sitting posture',' the moveability of the body on the seat' and the 'time sitting in a posture' as factors in the model. In their pilot experiment eleven subjects sat in an experimental chair for a 40 minute session. Two seat angles (10 and 20 degrees) and three seat cushion to back rest angles (95, 100 and 105 degrees) were used 
Gyi, D.E. and Porter J.M. (1999). Interface pressure and the prediction of car seat discomfort. Applied Ergonomics 30, 99-107.

in a random order to give six postures. General ratings of discomfort were not found to be sensitive to the postural differences but pressure measurements did significantly reflect these changes. Again the short duration of subjects in each posture (just five minutes) is of concern, as reported discomfort may vary considerably with time. Also there was no task specified for the subjects to carry out, which could change opinions as to their preferred posture. As Pile (1979) cited in Zacharkow (1988) pointed out, 'what is considered comfortable by a user depends very much on the way a seat is used and how long it is used'.

In summary, the literature regarding interface pressure measurement is limited. Only a few studies have attempted to correlate discomfort with pressure and the duration of such trials was usually only 5-10 minutes. However, simple relationships had already been established by some authors between pressure and body type (Garber and Krouskop, 1982), pressure and gender (Yang et al, 1984; Zacharkow, 1988; Sember III, 1994), pressure and seat hardness (Congleton et al, 1988; Riley and Bader, 1988;) and pressure and posture under controlled experimental conditions (Treaster and Marras, 1989; Shen and Galer, 1993). Further exploration of the technique was clearly required as it seemed that so far any conclusions from such studies were unclear and in some cases contradictory.

\section{Rationale for the experimental studies}

In order to identify robust relationships between interface pressure and reported discomfort with 'real world applicability', it was decided to undertake two experiments. The first experiment varied only foam hardness, whilst the second experiment changed only the driving posture. Consequent changes were observed in interface pressure and reports of discomfort.

The prediction of car occupant discomfort from the analysis of pressure distribution data should be comparable with actual reports of discomfort over a period of time that is representative of extended driving. The Vehicle Ergonomics Group (VEG) have been conducting road trials and evaluating car seats since 1981 and have found that at least two 
Gyi, D.E. and Porter J.M. (1999). Interface pressure and the prediction of car seat discomfort. Applied Ergonomics 30, 99-107.

hours is required to clearly differentiate between various seats and that the initial assessment of a seat is often a poor judgement of its longer duration performance. Figure 1 illustrates an example of a VEG road trial where 'car C' performed very well in terms of 'showroom comfort', having no discomfort after 15 minutes in the lower back and right buttock. Even after 45 minutes there was no reported discomfort in the right buttock. However, 'car C' was the most uncomfortable car after 135 minutes in the lower back and right thigh (Porter and Reed, 1992).

\section{Method}

A static, laboratory based experiment allowed a more controlled environment and therefore a highly adjustable driving rig was constructed (Figure 2). Seat height, steering wheel height, steering wheel distance from the body, pedal height and pedal distance from the body were all independently adjustable. The seat itself was also adjustable in tilt, backrest angle and lumbar support. The pedals, gearbox and steering wheel required realistic forces to operate. Two videos of the 2.5 hour (60 mile) test route used regularly by VEG in their road trials were made for use with the rig, giving a driver's view of the road, with a voice-over of instructions about the route to guide the driver when to change gear, slow down etc. The videos encompassed a range of road types including motorways, country roads and town driving for the simulation of driving tasks.

Seven experimental seats were also constructed for use with the rig, which were identical in all respects (colour, profile etc.) with the exception of foam density. The range of densities were within car seat production limits to simulate real world conditions of soft to hard car seats (Table 1). The method of paired comparisons, a psychological scaling method described in detail in Guilford (1954), was used to determine subject's preferred and least preferred seats for the trials. Briefly, the car seats were numbered and listed in all possible pairings. Subjects were then presented with the 21 pairings of the 7 car seats. For each seat they adjusted the backrest for comfort, mimicked driving and then made a choice as to their preferred seat. Subjects could test each seat as many times as they wished for each comparative judgement, but were advised not to deliberate too 
Gyi, D.E. and Porter J.M. (1999). Interface pressure and the prediction of car seat discomfort. Applied Ergonomics 30, 99-107.

long. A table was then constructed showing the proportion of occasions one car seat was preferred over another from which a ranking of the car seats was calculated.

Experimental sessions took place on separate days, but at the same time of day. At the start of each driving rig trial, subjects completed three questionnaires: a seat feature checklist for a detailed evaluation of the seat cushion, backrest and lumbar support; a seat detail questionnaire assessing seat hardness and support; and a body part comfort/discomfort chart (Figure 3) based on that of Corlett and Bishop (1976). Subjects completed a further 4 body part comfort/discomfort charts, each at 30 minute intervals. At the end of each trial the another seat detail questionnaire was completed.

Each subject's driving posture was measured after 60 minutes using anatomical landmarks and a goniometer. Postural angles recorded adapted from Grandjean et al (1983), Bridger (1988) and Bhatnager et al (1985) are shown in Table 2.

At the end of the experimental session a modified cell matrix was carefully positioned on the seat, and subjects assumed their driving posture. Interface pressure readings were taken using the modified cell matrix with the Talley Pressure Monitor (TPM) Mk III. The redesign of the TPM cell matrix is described in detail in Gyi et al (1998). Briefly, a half matrix was designed to measure pressure under the right side of the body. Observation of earlier data showed little asymmetry and many other authors supported this view e.g. Congleton et al (1988). Subjects wore clothing without heavy seams, buttons or pockets to ensure a minimal effect on the readings.

\section{Experiment 1}

The first experiment examined whether seat interface pressure data could be used to predict differences in reported discomfort between car seats with different foam densities. The sample consisted of seven males with a mean age of 40.7 years (SD 17.9) and seven females with a mean age of 42.86 (SD 11.9). They were carefully selected to represent a wide range of stature $(1475-1875 \mathrm{~mm})$. They sat in each of their preferred and least preferred seats (from the seven experimental seats) for two 2.5 hour static driving trials in their optimum posture and in a balanced order. Their optimum posture and position of 
Gyi, D.E. and Porter J.M. (1999). Interface pressure and the prediction of car seat discomfort. Applied Ergonomics 30, 99-107.

the controls was subjectively determined by the method of fitting trials and confirmed by a 10-15 minute driving simulation. Posture was held constant within subjects but not between subjects, as drivers do not adopt identical postures. It could be expected that there would be higher correlations with interface pressure if the subjects held identical postures but this was not considered to be a realistic situation

\section{Experiment 2}

In the second study seat design was held constant by using the most comfortable seat overall, as selected by the method of paired comparisons. Posture was varied both within subjects and between subjects, by comparing a limited adjustment driving package (taken from a well-known car) with a fully adjustable driving package. In this way posture would vary within realistic constraints. In an attempt to ensure that there were some differences in reported discomfort between the two conditions, 12 subjects were selected who represented extremes of body height. These were six tall males with a mean stature of $1939 \mathrm{~mm}$ (SD 84) and six short females with a mean stature of $1543 \mathrm{~mm}$ (SD 22).

They also represented the groups of individuals who may have problems with a standard driving package being close to or outside the normal range of design criteria. They were all university students and consequently their mean age was 21.0 (SD 1.95) years. The repeated measures experimental design was otherwise identical to experiment 1 .

\section{Analyses}

The extensive data exploration and analyses were performed using SPSS for Mackintosh Computers (Norusis, 1990). The complete results and analyses are presented in Gyi (1996).

For the statistical analyses, three variables were calculated from the 7 point comfort/discomfort scale for each of the right buttock, right thigh and low back: mean rating over the trial (intensity), rating after 135 minutes and total minutes of reported discomfort. The latter variable allocated reported discomfort (i.e. a rating of 5, 6 or 7), a weighting of 30 minutes for each of the five time periods, from which the mean number 
Gyi, D.E. and Porter J.M. (1999). Interface pressure and the prediction of car seat discomfort. Applied Ergonomics 30, 99-107.

of minutes of discomfort for each subject in each body area could then be calculated.

Discomfort in the IT and thigh areas refer to discomfort in the right side of the body for the purpose of the analyses.

Seven interface pressure variables (Table 3) were calculated for each of the whole seat, the right IT, right thigh and low back areas, for statistical analysis with the body part discomfort variables. The readings of 9 sensors (area $5000 \mathrm{~mm}^{2}$ ) were used to define the area of contact under the right IT; and 8 sensors (area $7250 \mathrm{~mm}^{2}$ ) to define a central rectangular area under the right thigh. The low back pressure variables were calculated from the sensors identified as the contact area of the low back. Pressure values throughout this paper are given in $\mathrm{mmHg}(1 \mathrm{mmHg}=133.322387 \mathrm{~Pa})$. In addition pressure values in the IT and thigh areas always refers to the right side of the body.

Parametric and non-parametric measures of linear association and sample differences were employed. Also, the technique of multiple regression analysis was used in an attempt to explore the variables important in predicting thigh, buttock and low back discomfort and interface pressure values.

\section{Results and discussion}

The results of experiments 1 and 2 indicate that the simple quantification of interface pressure data from a variety of individuals, with the assumption that high (or low) pressure values are predictors of increased discomfort, is unsatisfactory. The four contour pressure maps in Figure 4 (of different subjects) have been selected to illustrate this lack of clarity. Plots 1 and 2 illustrate markedly different pressure distributions; a mean ischial tuberosity (IT) pressure of $106.7 \mathrm{mmHg}$ (SD 40.9) and a mean thigh area pressure of $23.4 \mathrm{mmHg}$ (SD 14.8) in plot 1, compared with a mean IT pressure of 36.6 $\mathrm{mmHg}(\mathrm{SD} 10.35)$ and a mean thigh area pressure of $32.4 \mathrm{mmHg}$ (SD 1.58) in plot 2. Despite these differences, both drivers reported the same amount of discomfort i.e. 120 minutes of buttock discomfort and 90 minutes of thigh discomfort during the 150 minute session. 
Gyi, D.E. and Porter J.M. (1999). Interface pressure and the prediction of car seat discomfort. Applied Ergonomics 30, 99-107.

Plots 3 and 4 also show two very different distributions; a mean IT pressure of 55.2 $\mathrm{mmHg}(\mathrm{SD} 22.6)$ and a mean thigh area pressure of $28.8 \mathrm{mmHg}(\mathrm{SD} 4.4)$ in plot 3, compared with a mean IT area pressure of $40.8 \mathrm{mmHg}$ (SD 1.58) and a mean thigh area pressure of $60.4 \mathrm{mmHg}$ (SD 14.8) in plot 4. However, neither of these drivers reported any discomfort in the buttocks or thighs.

These data show the difficulties of benchmarking ranges of pressure for comfort or discomfort. Such inconsistencies in interface pressure data are now further illustrated.

\section{Experiment 1}

It was expected that the least preferred seat (seat 1, for 11 subjects) would cause more discomfort over time. Figure 5a for example, shows that this was generally the case for males with significant differences $(p<0.05)$ between the two seats for the total minutes of reported discomfort in the buttocks and thigh areas. However, for the females there no significant differences in the total number of minutes of reported discomfort, despite obvious differences in foam hardness (Figure 5b).

Figures $5 \mathrm{c}$ and $\mathrm{d}$ represent graphically the mean ischial tuberosity (IT), thigh and low back area pressures, as examples of some of the descriptive data for seat pressure. The mean pressure values were selected as being the most stable and least influenced by measurement error. No significant differences were found in mean IT, thigh and low back area pressures between the two conditions for either the complete sample or gender, once again despite differences in foam hardness. Although Figures $5 \mathrm{c}$ and $\mathrm{d}$ show a trend for higher mean pressures with the least preferred (often harder) seat, the ranges of differences in the means were small, 1-4 mmHg (females) and 2-7 mmHg (males).

Using Spearman's rank order correlation, the only consistent relationship between reported discomfort and any of the pressure variables in experiment 1 was for females, with their preferred seat. In this case, significant negative correlations were found between pressure and discomfort variables for the low back, i.e. reports of discomfort were less as pressure increased. Reported discomfort over time was the same for both seats, but there was a surprising trend (not significant) for females to report more total 
Gyi, D.E. and Porter J.M. (1999). Interface pressure and the prediction of car seat discomfort. Applied Ergonomics 30, 99-107.

minutes of discomfort with their preferred seat (Figure 5b). It could be that the mean low back pressure for their preferred seat of $23 \mathrm{mmHg}$ (range 19-29) was too low for adequate support, although this mean value was only $3 \mathrm{~mm} \mathrm{Hg}$ lower than the mean for the least preferred seat (Figure 5d).

\section{Experiment 2}

Generally, there was more reported discomfort than experiment 1, especially with the sample of tall males (Figure 6a). For example, the mean total minutes of reported discomfort for males in the low back was 110 minutes with the limited package compared with 80 minutes with the fully adjustable package. As with experiment 1 , there was little difference in the frequency of reported discomfort in the females between the two driving packages (Figure 6b). The tall males however, were forced into a constrained posture by the limited driving package and consequently reported more discomfort at the different time periods and in more body areas (significant or approaching significance in the neck; upper, mid and low back; left and right buttock, arm, foot and ankle; and left knee).

Referring to Figure $6 \mathrm{c}$, it can be seen that there was a large and significant difference $(p<0.05)$ in the mean IT area pressure for the tall males between the two driving packages $(75.7 \mathrm{mmHg}$ with the limited package, compared with $53.7 \mathrm{mmHg}$ for the fully adjustable package). Consistent, significant correlations were found for the sample of tall males between the buttock discomfort and IT area pressure variables for both driving packages, but less so for the short females. Also, in the multiple regression analysis, for tall males using the limited driving package, the variable 'buttock discomfort after 135 minutes' was selected along with 'sitting height' and 'hip breadth' as a significant predictor of mean IT area pressure explaining $99 \%$ of the variance.

Although IT area pressures for the short females with the limited driving package were significantly higher than those with the fully adjustable package (Figure 6d), these were not reflected in significantly more reported buttock discomfort, unlike for the sample of males. The mean IT area pressure experienced with the limited driving package by the males was $76 \mathrm{mmHg}$ compared to only $44 \mathrm{mmHg}$ for the females. This high IT area 
Gyi, D.E. and Porter J.M. (1999). Interface pressure and the prediction of car seat discomfort. Applied Ergonomics 30, 99-107.

pressure in the tall males was a consequence of the limited legroom which necessitated a 'knees up' flexed posture.

Similar pressures were found under the thighs between the two packages for both the males and females (Figures $6 \mathrm{c}$ and d). Also, neither sex had significant differences in reported thigh discomfort.

The frequencies of reported discomfort in the low back for both tall males and the short females were generally high (Figure $6 \mathrm{a}$ and $\mathrm{b}$ ), but no consistent correlations between the back discomfort and low back pressure variables were found.

\section{Discussion of both experiments}

The difficulties of benchmarking levels of pressure for the prediction of comfort/discomfort, is well illustrated by the low back data from the two experiments. In experiment 1, the mean low back pressure for males with their preferred seat was 29 $\mathrm{mmHg}$ and there was little reported low back discomfort i.e. only one male reported some discomfort at 135 minutes. However despite a mean low back pressure of $29 \mathrm{mmHg}$ in males with the fully adjustable driving package in experiment 2, 33\% of them reported discomfort in the low back after 15 minutes rising to $67 \%$ after 135 minutes.

These difficulties continue even if males and females are considered separately. In males, there was a trend for lower mean IT pressures to be associated with reported discomfort in experiment 1, whereas higher mean IT pressures were associated with reported discomfort in experiment 2. Similar inconsistencies can be seen with the thigh and low back data, and also the data for the females.

Direct comparison of the pressure values obtained in these experiments with those in the literature is difficult because of the different experimental conditions or lack of information on these conditions (e.g. body type, seat surface, task carried out and pressure measurement technologies). Kurz et al (1989) actually make recommendations for ergonomic vehicle seat design, for pressures directly under IT to be $75-225 \mathrm{mmHg}$ and immediately around the IT to be $60-113 \mathrm{mmHg}$. Unfortunately, it is not known if these are maximum or mean values and no method or reference is given, but they are 
Gyi, D.E. and Porter J.M. (1999). Interface pressure and the prediction of car seat discomfort. Applied Ergonomics 30, 99-107.

clearly higher than the values found in experiments 1 and 2. Sember III (1994) advises that the maximum pressure that can be sustained under the IT's without discomfort after 15 minutes is $62 \mathrm{mmHg}$ for men under 30 and women under 40 . He then goes on to advise that IT pressures of $26 \mathrm{mmHg}$ for the over 40 's and $15 \mathrm{mmHg}$ for the elderly, will produce discomfort.

Kamijo et al (1982) recommended mean pressures of 11-18 mmHg for supporting the lumbar area in car seats, although only one male subject was used in their experiments. This is lower than any of the mean pressures in the low back for both experiments 1 and 2. For example, once again referring to Figures $5 c-d$ and $6 c-d$, the range of mean low back pressures was 21-35 mmHg.

For both experiments, the subjects' predictions (at the beginning of the session) and observations (at the end of the session) of seat hardness were not consistent with areas of higher pressures or more reported discomfort. It seems that the subjective judgement of seat hardness in the IT, thigh and low back areas, did not relate to the body areas in which discomfort was actually experienced. It may be that 'trained subjects' are required to make these 'expert judgements'. Perhaps subjective judgements of 'seat pressure' or 'areas of the seat causing discomfort' rather than ' seat hardness' may have revealed more significant outcomes.

\section{Other factors affecting interface pressure values}

In agreement with Yang et al (1984), for both experiments the males generally had higher pressures values than females, significantly so under the IT area (experiments 1 and 2, both conditions) and the thigh area (experiment 2, both packages). The variables of 'sex' and 'hip breadth' (which is probably sex related) were also selected by the multiple regression analysis as being the best predictors of mean IT pressure in experiment 1 . Zacharkow (1988) suggested that males may exhibit higher pressures as they have less subcutaneous fat in the buttocks and hips, are more heavily built above the pelvis and the fact that the IT's and the acetabula (the sockets for the head of the femur) are closer together with the ischia being more inverted in shape. This could account for the higher 
Gyi, D.E. and Porter J.M. (1999). Interface pressure and the prediction of car seat discomfort. Applied Ergonomics 30, 99-107.

sensitivity of the males to the harder seat (experiment 1) and to the constrained posture (experiment 2).

The Reciprocal Ponder Index or RPI (Yang et al, 1984) was used as a measurement of a persons body build relative to others. It is calculated by dividing body length in centimetres by the cubed root of body weight in kilograms. A high value indicates a narrow, thin body and a low value indicates a wide body build. Consistent with the findings of Garber and Krouskop (1982), thinner subjects had higher IT pressures, although Yang et al (1984) failed to find such a relationship in their study. The sample of tall males in experiment 2, who had the highest IT pressures also were generally thinner than the other sample groups. For example, the mean RPI for these males was 47.4 (SD 5.5) compared with 41.5 (SD 1.3) for the short females, 42.4 (SD 1.6) for the more varied group of males in experiment 1 and 39.9 (SD 2.2) for the females in experiment 1 . In both experiments significant positive correlations were also found between weight and thigh pressure values, although once again this is in disagreement with Yang et al (1984). It is proposed that the lack of significant findings in the study by Yang et al (1984) could be due to their small ranges of height (1450-1720 $\mathrm{mm}$ ) and weight (42-79 $\mathrm{kg}$ ) or maybe even differences in the body build of the Chinese subject group. Very few of the studies, considered gender or differences in body build when reporting their findings.

Bennett et al (1979) concluded from their experiments that although pressure is the primary force, the pressure required to produce capillary occlusion could be halved when accompanied by sufficient shear. The studies described in this paper did not record shear forces at the seat / occupant interface. It is hoped that future pressure sensing systems will allow such information to be collected and that the more detailed data will aid the identification of a clearer relationship between interface pressure and reported discomfort.

\section{Conclusions}

1. Despite the automotive industries' interest in interface pressure measurement, critical appraisal of the literature does not support the finding of a simple relationship between 
Gyi, D.E. and Porter J.M. (1999). Interface pressure and the prediction of car seat discomfort. Applied Ergonomics 30, 99-107.

discomfort and pressure. Those studies supporting such a relationship provided few details of their experimental design and data analysis. They were also often based on short-term discomfort evaluations or had a small number of subjects.

2. The research reported in this paper has demonstrated that the simple quantification of interface pressure data from a variety of individuals, with the assumption that high, or even low pressure values (in the case of the low back), are predictors of increased discomfort is unsatisfactory. It seems that this technique is not robust enough to provide such information to the automotive industry in 'real world situations' i.e. to include a large variety of occupants (male, female, body builds, ages) with different car seat designs and chosen postures.

3. Body build appears to have a significant influence on pressure values in the IT and thigh areas, such that thinner subjects (high RPI) had higher pressures in the IT area and heavier subjects (weight) had higher pressures under the thigh.

4. Although there were consistent, significant relationships between IT pressure values and IT discomfort variables, these were only for the sample of very tall males (95th percentile stature and above) with the limited adjustment driving package in experiment

2. These males would be outside the anthropometric dimensions considered by many designers working in the automotive industry. Due to the constrained postures imposed by many vehicles they could also be expected to experience discomfort more frequently than other car users. The fact that pressure values could only potentially be used as predictors of IT discomfort with this 'extreme' sample, casts strong reservations concerning the use of interface pressure measurement as a predictive technique for discomfort in the automotive industry.

\section{Acknowledgements}

The authors would like to acknowledge the Brite-Euram European Initiative (Project 5547) who funded this research. Loughborough University was one of several European based partners in the consortium (which also included car manufacturers and seat designers) whose joint objective was to improve car seat design. 
Gyi, D.E. and Porter J.M. (1999). Interface pressure and the prediction of car seat discomfort. Applied Ergonomics 30, 99-107.

\section{References}

Bennett, L., Kavner, D., Lee, B.K. and Trainor, F.A. (1979) 'Shear vs pressure as causative factors in skin blood flow occlusion' Archives of Physical Medicine and Rehabilitation, 60, 309-314.

Bhatnager, V., Drury, C.G. and Schiro, S.G. (1985) 'Posture, postural discomfort and performance' Human Factors, 27 (2), 189-199.

Bridger, R.S. (1988) 'Postural adaptions to a sloping chair and work surface' Human Factors, 30 (2), 237-247.

Congleton, J.J., Ayoub, M.M. and Smith, J.L. (1988) 'The determination of pressures and patterns for the male buttocks and thigh in sitting utilizing conductive foam’ International Journal of Industrial Ergonomics, 2, 193-202.

Corlett, E.N. and Bishop, R.P. (1976) 'A technique for assessing postural discomfort' Ergonomics, 19 (2), 175-182.

Garber, S.L. and Krouskop, T.A. (1982) 'Body build and its relationship to pressure distribution in the seated wheelchair patient' Archives of Physical Medicine and Rehabilitation, 63, 17-20.

Grandjean, E., Hunting, W. and Pidermann, M. (1983) 'VDT and workstation design: preferred settings and their effects' Human Factors, 25, 161-175.

Gross, C.M., Goonetilleke, R.S., Menon, K.K., Banaag, J.C.N. and Nair, C.M. (1994) In R. Lueder and K. Noro, eds.: Hard Facts about Soft Machines: The Ergonomics of Seating, 231-253. London: Taylor \& Francis.

Guilford, J.P. (1954) Psychometric Methods, London: McGraw-Hill Book Company, Inc.

Gyi, D.E. (1996) Driver discomfort: Prevalence, prediction and prevention, $\mathrm{PhD}$ Thesis, Loughborough University.

Gyi, D.E., Porter, J.M. and Robertson, N.K.B. (1998) 'Seat pressure measurement technologies: Considerations for their evaluation' Applied Ergonomics, 27 (2), 85-91. 
Gyi, D.E. and Porter J.M. (1999). Interface pressure and the prediction of car seat discomfort. Applied Ergonomics 30, 99-107.

Kamijo, K., Tsujimura, H., Obara, H. and Katsumata, M. (1982) 'Evaluation of seating comfort' SAE Technical Paper No. 820761.

Kurz, B., Diebschlag, W. and Heidinger, F. (1989) 'Recommendations for ergonomic and climatic physiological vehicle seat design' Journal of Cellular Plastics, 25, 125-137.

Lee, J. and Ferraiuolo, P. (1993) 'Seat comfort' S.A.E. Technical Paper No. 930105. Norusis, M.J. (1990) SPSS Base System User's Guide Chicago, USA: SPSS Inc. Pile, J.F. (1979) Modern Furniture. New York: John Wiley and Sons.

Porter, J.M. (1995) 'The ergonomics development of the Fiat Punto European car of the year' In A. de Moraes and S. Marino (eds).: Proceedings of the International Ergonomics Association World Conference 1995, 73-76, Rio de Janeiro.

Porter, J.M. and Read, T. (1992) 'Road trial evaluation of Fiat prototype cars, Honda Civic 1.6 DX, and Renault Clio 1.4 RT' Vehicle Ergonomics Group, Loughborough University of Technology (unpublished and confidential report).

Riley, S. and Bader, D.L. (1988) 'Biomechanical measurements of back shape and interface pressures in unsupported sitting' Clinical Biomechanics, 3, 114-117.

Sember III, J.A. (1994) 'The biomechanical relationship of seat design to the human anatomy' In R. Lueder and K. Noro, eds.: Hard Facts about Soft Machines: The Ergonomics of Seating, 221-229. London: Taylor \& Francis.

Shen, W. and Galer, I.A.R. (1993) 'Development of a pressure related assessment model of seating comfort' Proceedings of the Human Factors and Ergonomics Society 37th Annual Meeting, October 1993, (2), 831-835.

Treaster, D. and Marras, W.S. (1989) 'Seat pressure: measurement and analysis' S.A.E Technical Paper No. 890849.

Yang, B.J., Chen, C.F., Lin, Y.H. and Lien, I.N. (1984) 'Pressure measurement on the ischial tuberosity of the human body in sitting position and evaluation of the pressure relieving effect of various cushions' Journal of Formosan Medical Association, 83, 682698.

Zarcharkow, D. (1988) Posture: Sitting, Standing, Chair Design and Exercise.

Illinois: Charles C. Thomas Publisher. 
Gyi, D.E. and Porter J.M. (1999). Interface pressure and the prediction of car seat discomfort. Applied Ergonomics 30, 99-107. 
Gyi, D.E. and Porter J.M. (1999). Interface pressure and the prediction of car seat discomfort. Applied Ergonomics 30, 99-107.

Table 1. Detail regarding the seat foams in the experimental seats. NB Std (standard) refers to the production hardness for the seat.

\begin{tabular}{llll}
\hline & Description of car seat hardness & $\begin{array}{l}\text { Cushion } \\
(\mathbf{d a N})\end{array}$ & $\begin{array}{l}\text { Backrest } \\
(\mathbf{d a N})\end{array}$ \\
\hline 1 & Hard cushion; hard backrest & 48.4 & 36.6 \\
2 & Std cushion back \& hard cushion front; std backrest & $36.0 \& 50.0$ & 18.0 \\
3 & Std cushion back \& soft cushion front; std backrest & $37.0 \& 20.0$ & 18.0 \\
4 & Medium soft cushion; medium soft backrest & 32 & 17 \\
5 & Soft cushion; medium soft backrest & 27.2 & 17 \\
6 & Medium hard cushion; medium hard backrest & 42.8 & 25.2 \\
7 & Std cushion; std backrest & 37.0 & 18.0 \\
\hline
\end{tabular}


Gyi, D.E. and Porter J.M. (1999). Interface pressure and the prediction of car seat discomfort. Applied Ergonomics 30, 99-107.

Table 2. Definition of postural angles, adapted from Grandjean et al (1983), Bridger (1988) and Bhatnager et al (1985).

\begin{tabular}{|c|c|}
\hline Postural angle & Definition \\
\hline Neck inclination & $\begin{array}{l}\text { The angle between the vertical and a line from the } 7^{\text {th }} \\
\text { cervical vertebrae to the auditory canal }\end{array}$ \\
\hline Trunk-thigh angle & $\begin{array}{l}\text { The angle between a line from the acromium to the } \\
\text { greater trochanter and a line from the lateral condyle to } \\
\text { the greater trochanter }\end{array}$ \\
\hline Arm flexion & $\begin{array}{l}\text { The angle between the vertical and a line from the } \\
\text { acromium to the lateral epicondyle }\end{array}$ \\
\hline Elbow angle & $\begin{array}{l}\text { The angle between a line from the acromium to the lateral } \\
\text { epicondyle and a line from the ulnar styloid to the lateral } \\
\text { epicondyle }\end{array}$ \\
\hline Knee angle & $\begin{array}{l}\text { The angle between a line from the greater tronchanter to } \\
\text { the lateral condyle and a line from the lateral malleolus to } \\
\text { the lateral condyle }\end{array}$ \\
\hline Ankle angle & $\begin{array}{l}\text { The angle between a line from the lateral condyle to the } \\
\text { lateral malleolus and a line parallel with the foot. }\end{array}$ \\
\hline
\end{tabular}


Gyi, D.E. and Porter J.M. (1999). Interface pressure and the prediction of car seat discomfort. Applied Ergonomics 30, 99-107.

Table 3. The interface pressure values calculated.

\begin{tabular}{ll}
\hline Variable & Description \\
\hline Average seat ratio & Ratio between seat mean and back mean \\
Maximum & Maximum pressure value in the defined area \\
Mean & Mean pressure in defined area \\
Standard deviation (SD) & SD from the mean in defined area \\
Total & Sum of the cells in defined area \\
Ratio maximum & Minimum/maximum in defined area \\
Ratio minimum & Second highest pressure/maximum in defined area \\
Proportion & 'Total'/seat or back total \\
\hline
\end{tabular}


Gyi, D.E. and Porter J.M. (1999). Interface pressure and the prediction of car seat discomfort. Applied Ergonomics 30, 99-107.

Fig 1. The percentage of subjects reporting discomfort in four comparable cars (adapted from Porter and Reed, 1992).

low back

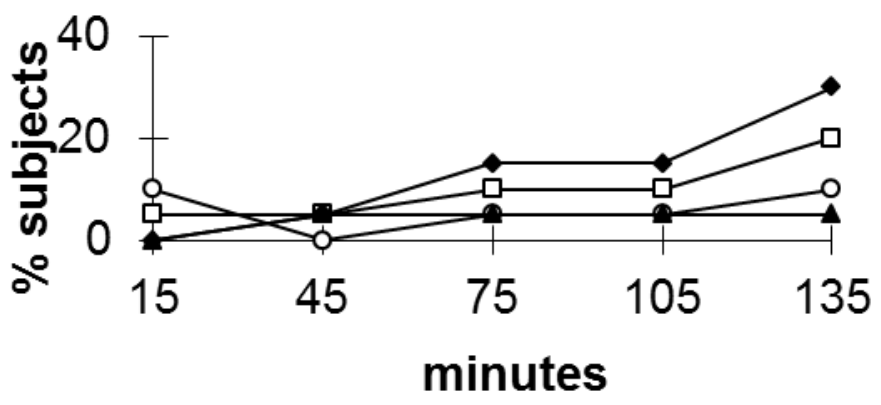

right buttock

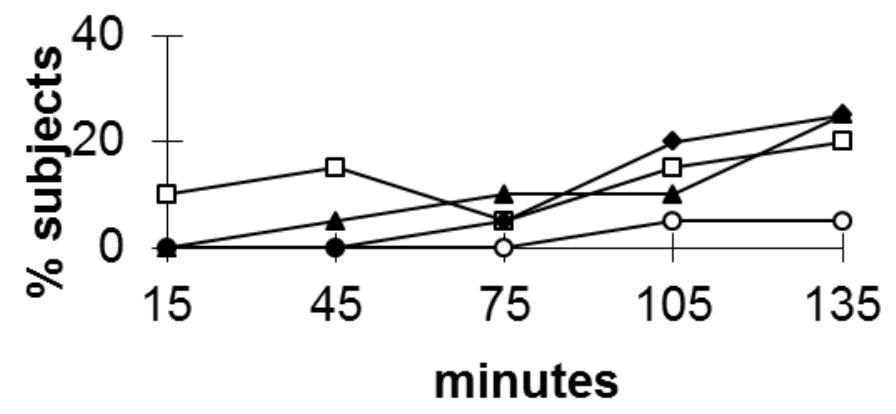

right thigh

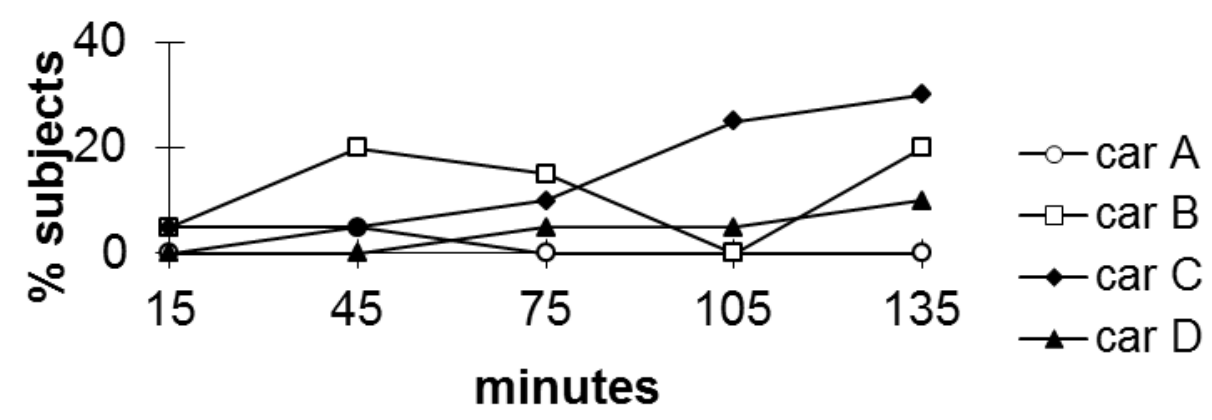


Gyi, D.E. and Porter J.M. (1999). Interface pressure and the prediction of car seat discomfort. Applied Ergonomics 30, 99-107.

Fig 2. The experimental driving rig.

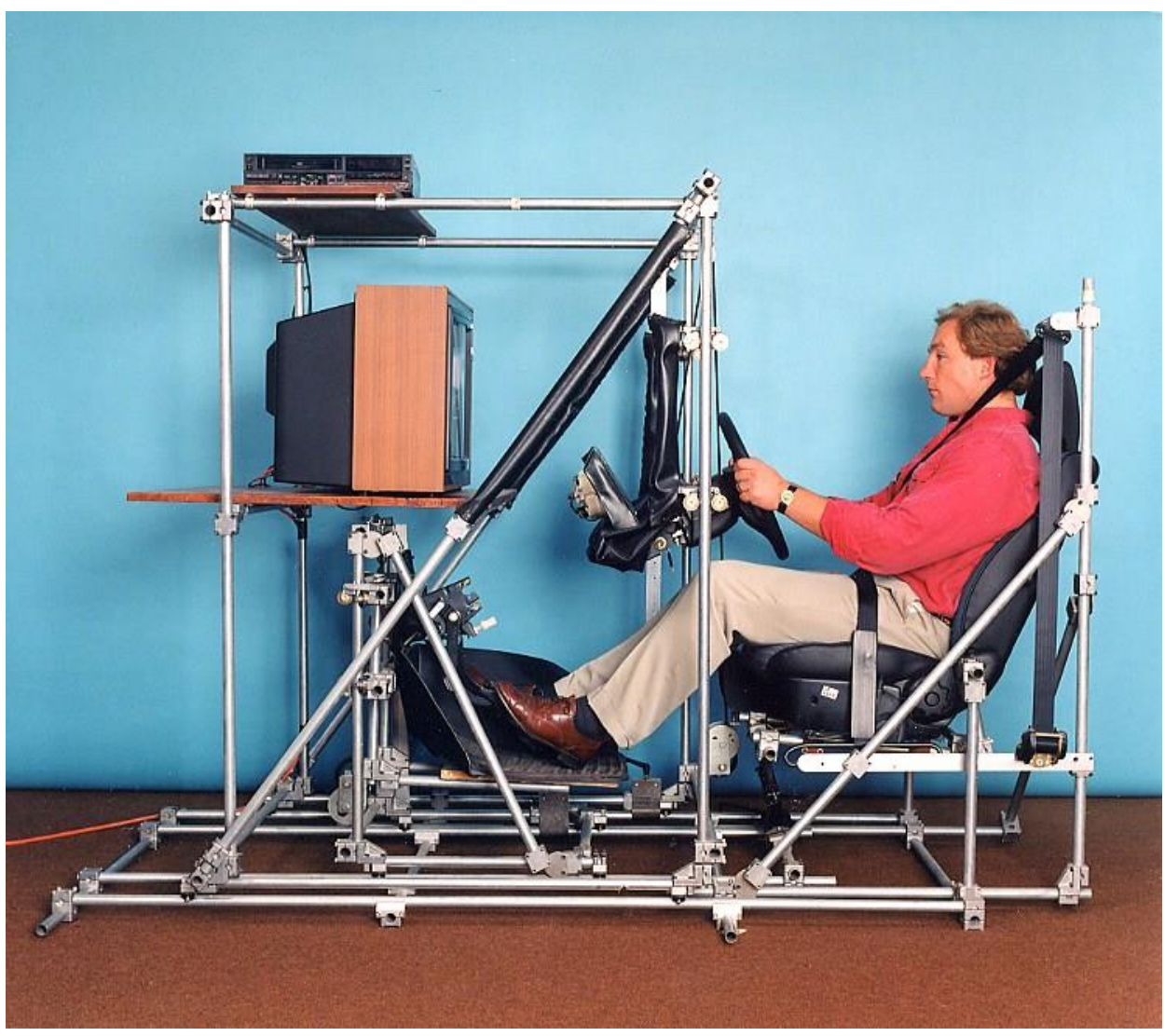


Gyi, D.E. and Porter J.M. (1999). Interface pressure and the prediction of car seat discomfort. Applied Ergonomics 30, 99-107.

Fig 3. Body part discomfort chart (based on Corlett and Bishop, 1976); 1. Very comfortable; 2. Moderately comfortable; 3 . Fairly comfortable; 4. Neutral; 5. Slightly uncomfortable; 6. Moderately uncomfortable; 7. Very uncomfortable.

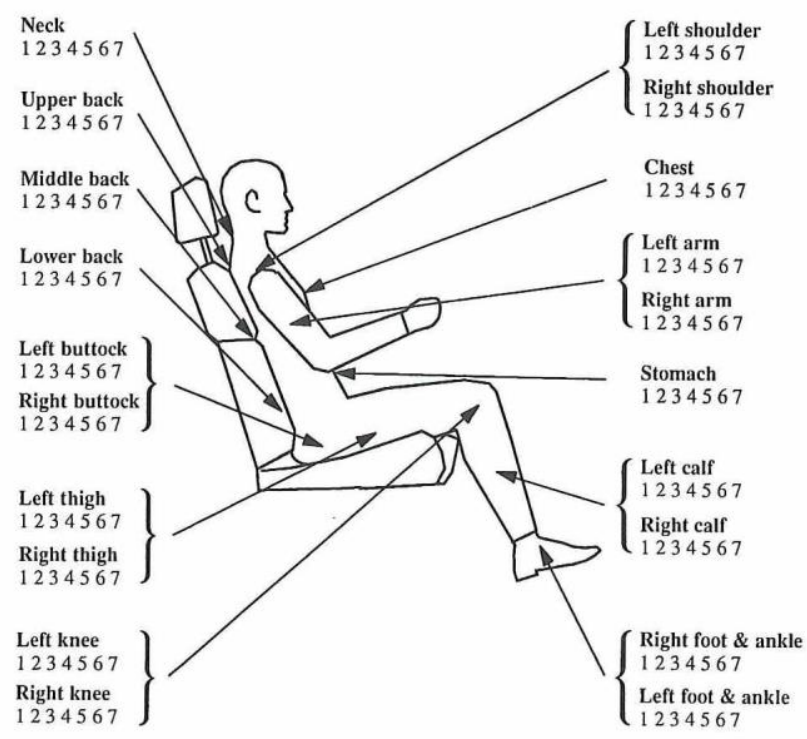


Gyi, D.E. and Porter J.M. (1999). Interface pressure and the prediction of car seat discomfort. Applied Ergonomics 30, 99-107.

Fig 4. Pressure distribution plots for the seat cushion area.
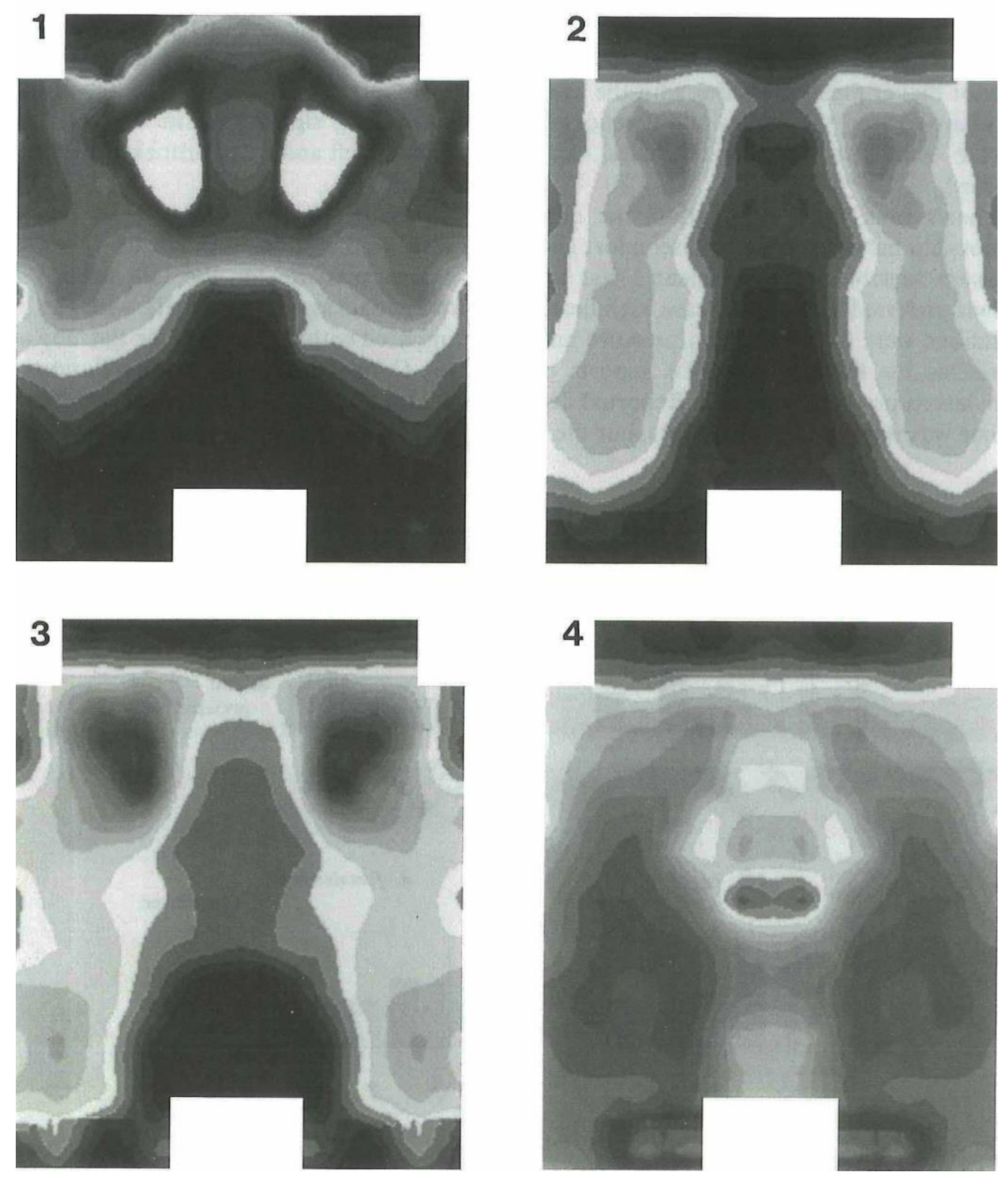
Gyi, D.E. and Porter J.M. (1999). Interface pressure and the prediction of car seat discomfort. Applied Ergonomics 30, 99-107.

Fig 5. Graphs illustrating the mean minutes discomfort and mean pressure values for males and females with their preferred and least preferred seats (expt. 1).

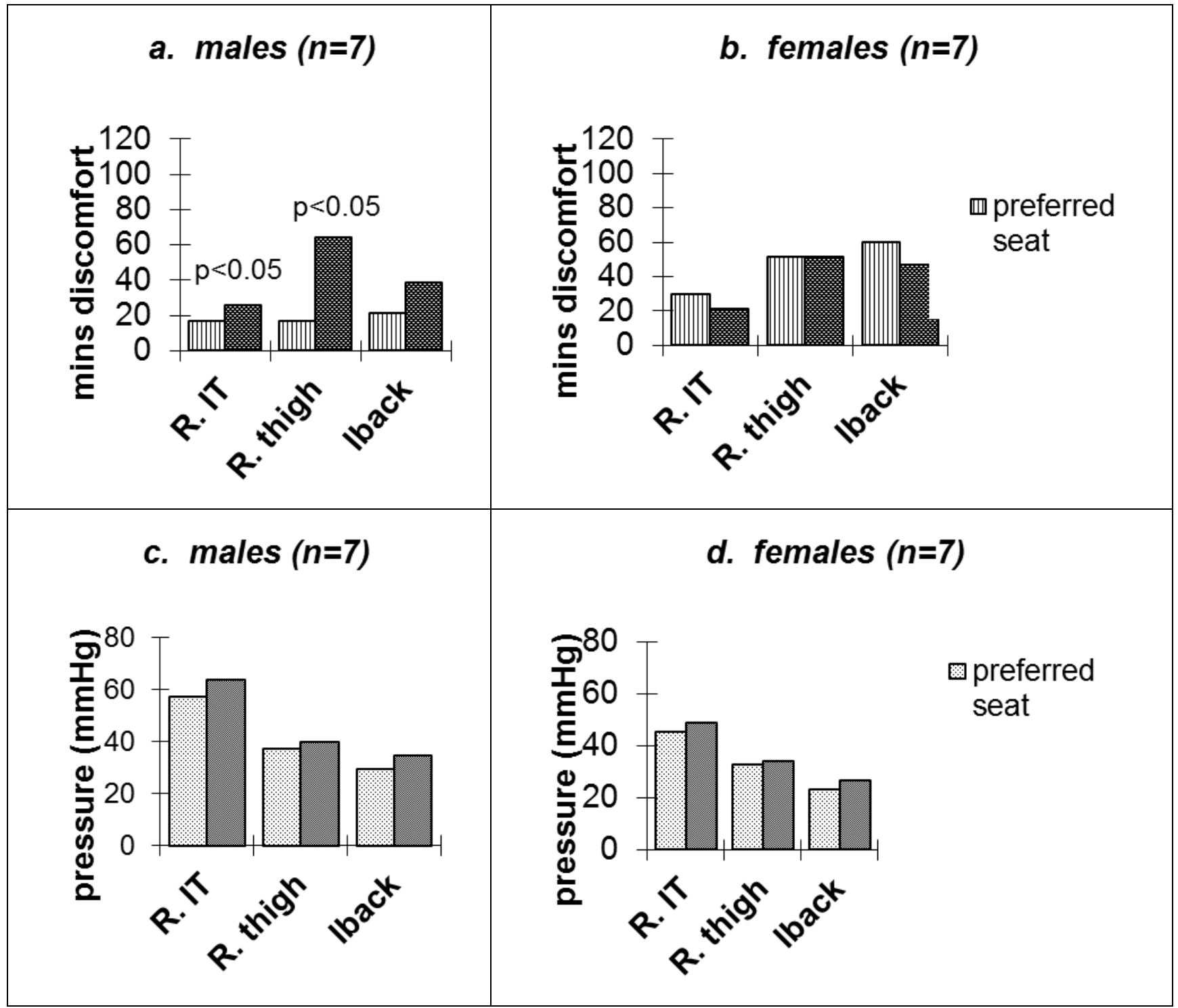


Gyi, D.E. and Porter J.M. (1999). Interface pressure and the prediction of car seat discomfort. Applied Ergonomics 30, 99-107.

Fig 6. Graphs illustrating the mean minutes discomfort and mean pressure values for males and females with the fully adjustable and limited driving packages (expt. 2).

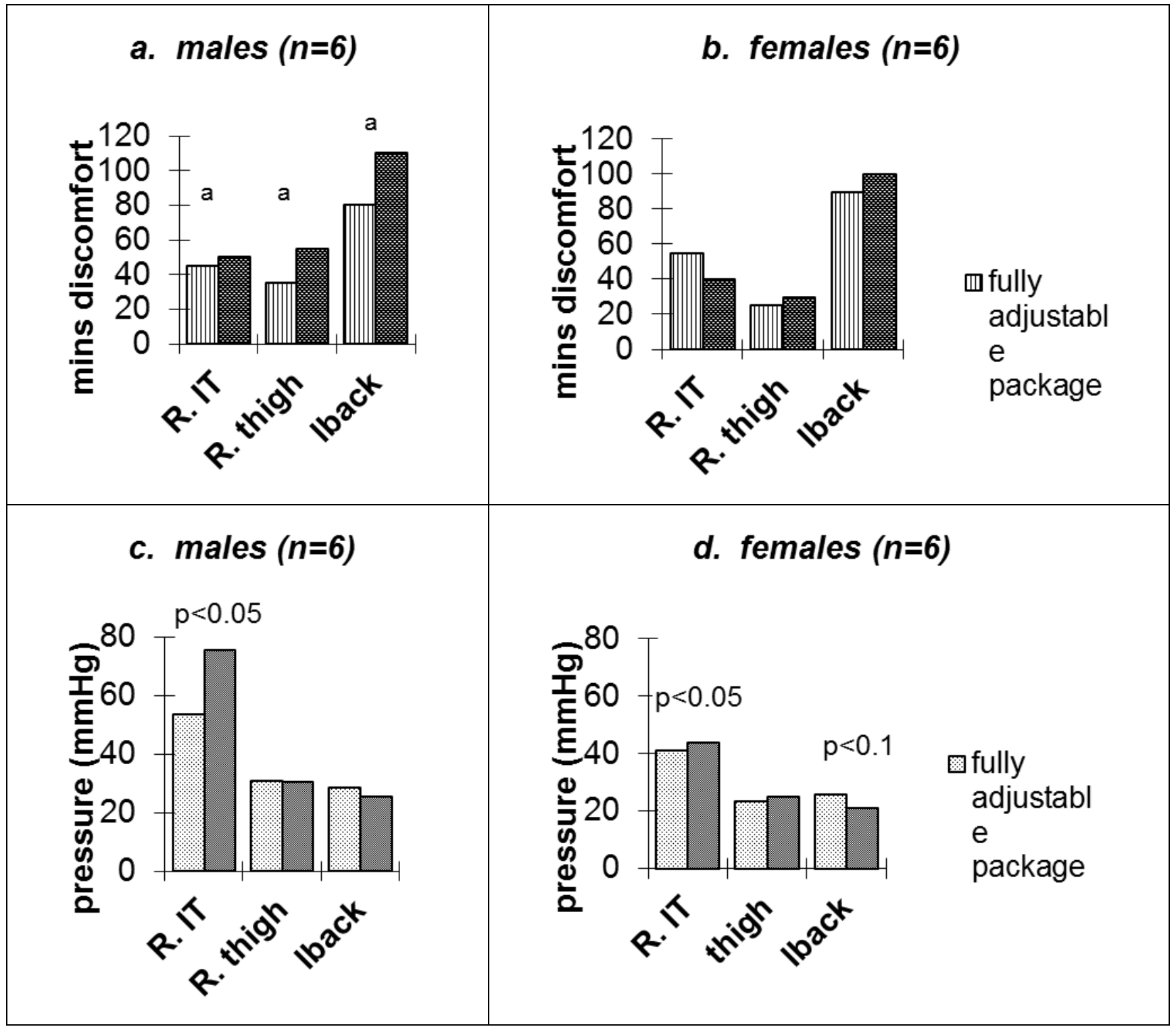

